PROCEEDINGS OF THE

AMERICAN MATHEMATICAL SOCIETY

Volume 130, Number 11, Pages 3335-3347

S 0002-9939(02)06678-9

Article electronically published on May 29, 2002

\title{
POLYNOMIAL INTERPOLATION, IDEALS AND APPROXIMATION ORDER OF MULTIVARIATE REFINABLE FUNCTIONS
}

\author{
THOMAS SAUER \\ (Communicated by David R. Larson) \\ Dedicated to C. A. Micchelli on the occasion of his 60th birthday, \\ with friendship and gratitude for a wonderful collaboration.
}

\begin{abstract}
The paper identifies the multivariate analog of factorization properties of univariate masks for compactly supported refinable functions, that is, the "zero at $\pi$ "-property, as containment of the mask polynomial in an appropriate quotient ideal. In addition, some of these quotient ideals are given explicitly.
\end{abstract}

\section{INTRODUCTION}

A function $f: \mathbb{R}^{s} \rightarrow \mathbb{R}$ is said to be refinable with respect to a mask $a=$ $\left(a_{\alpha}: \alpha \in \mathbb{Z}^{s}\right)$ if it satisfies a refinement equation of the form

$$
\phi=\sum_{\alpha \in \mathbb{Z}^{s}} a_{\alpha} \phi(2 \cdot-\alpha) .
$$

Due to the importance of refinable functions, in particular in connection with wavelet analysis, such functions are well studied, especially in one variable; cf. [7]. One point of interest in the research on refinable functions is the description of properties of the function $\phi$, for example smoothness, in terms of the mask vector $a$; cf. 4, 6]. A crucial property in this context is whether the algebraic span of the multi-integer translates of $\phi$ contains all polynomials of a certain degree, that is, whether

$$
\Pi_{n}(\mathbb{R})=\mathbb{S}(\phi):=\left\{\sum_{\alpha \in \mathbb{Z}^{s}} c_{\alpha} \phi(\cdot-\alpha): c_{\alpha} \in \mathbb{R}\right\},
$$

where $\Pi_{n}(\mathbb{R})$ denotes the vector space of all polynomials of total degree at most $n$ with real coefficients. This polynomial containment is necessary for $(n-1)-$ fold differentiability of $\phi$ (cf. [4]), and necessary and sufficient for $\phi$ providing

Received by the editors October 26, 2000 and, in revised form, June 19, 2001.

2000 Mathematics Subject Classification. Primary 42C40, 13P10; Secondary 41A05.

Key words and phrases. Subdivision, polynomial preservation, refinable functions, quotient ideals.

This work was supported by Deutsche Forschungsgemeinschaft with a Heisenberg fellowship, Grant \# Sa 627/6-1.

(C)2002 American Mathematical Society 
approximation order $n$. For further details, let me refer to the recent survey [9] and the references therein.

Here, we focus on the case that the mask $a$ or, equivalently, the refinable function $\phi$, are compactly supported. In this case, it is convenient to look at the symbol

$$
a(z)=\sum_{\alpha \in \mathbb{Z}^{s}} a_{\alpha} z^{\alpha}, \quad z \in \mathbb{C}^{s} \backslash\{0\},
$$

which is a Laurent polynomial, but after shifting the mask appropriately we might as well assume that $a(z)$ is a polynomial, that is, $a(z) \in \Pi:=\mathbb{C}\left[z_{1}, \ldots, z_{s}\right]$. In the univariate case it is well-known that containment of polynomials in $\mathbb{S}(\phi)$ can be related to factorizations of $a$ of the form

$$
a(z)=\frac{(1+z)^{n}}{2^{n}} b(z), \quad z \in \mathbb{C} .
$$

These factorizations are a handy tool for the construction of smooth refinable functions or for the "smoothing" of refinable functions, respectively, by taking a "reasonable" mask $b$ and multiplying it with factors of the form $(1+z) / 2$.

A popular statement about the multivariate case, however, is that factorization techniques cannot be extended from the univariate case since (in general) polynomials in more than one variable cannot be factored into linear polynomials. Of course, this statement is true but, nevertheless, there is a natural generalization of factorizations based on the notion of a quotient ideal. In fact, this paper will give an ideal theoretic characterization of the "sum rule"

$$
(q(D) a)(z)=0, \quad q \in \Pi, \operatorname{deg} q \leq n, \quad z \in\{-1,1\}^{s} \backslash\{(1, \ldots, 1)\},
$$

which is an equivalent description of (1.2). Having this description available, one can compute a basis for the ideal of solutions of (1.5), for example by using some computer algebra program, which then allows for a parametrization of all masks which satisfy (1.5) and hence for a systematic search for masks which admit refinable functions of a prescribed approximation order. However, it is worthwhile to mention that this algebraic condition does not guarantee the existence of the refinable function $\phi$, for example in $L_{2}\left(\mathbb{R}^{s}\right)$. To ensure this, additional properties on $a$ are necessary which can be expressed by the normalization property

$$
a(1, \ldots, 1)=2^{s},
$$

and, for example, in terms of the joint spectral radius of certain matrices; cf. 8].

The paper is organized as follows. In Section 2 we will review some basic facts on ideal bases and the connection to multivariate polynomial interpolation. Based on a characterization of particular ideal bases from this section, we will describe the ideal of all solutions of (1.5) in Section 3 and give explicit representations of this ideal in special cases in Section 4 .

\section{Polynomial interpolation And ideal theory}

In this section we recall and extend some results on the intimate connection between polynomial interpolation, polynomial ideal theory and ideal bases. A finite set $\Theta \subset \Pi^{\prime}$ of linearly independent functionals is called an ideal interpolation scheme [1] if the vector space

$$
\operatorname{ker} \Theta=\{f \in \Pi: \Theta(f)=0\} \subset \Pi
$$


is an ideal in $\Pi$, denoted by $\mathcal{I}_{\Theta}$. This is the case if and only if the functionals in $\Theta$ consist of consecutive derivatives evaluated at given points; cf. [3, 11].

To study the ideal $\mathcal{I}_{\Theta}$ more carefully, we recall the notion of grading: an additive monoid $\Gamma$, equipped with a well-ordering " $<$ ", is called a grading monoid if there is a direct sum decomposition

$$
\Pi=\bigoplus_{\gamma \in \Gamma} \Pi_{\gamma}
$$

such that each homogeneous subspace $\Pi_{\gamma}$ is a $\mathbb{C}$-vector space and such that $\Pi_{\gamma} \Pi_{\gamma^{\prime}} \subseteq$ $\Pi_{\gamma+\gamma^{\prime}}$. The two "standard" gradings are the $\mathrm{G}$ ("Gröbner") gradings, where $\Gamma=\mathbb{N}_{0}^{s}$ equipped with a term order and $\Pi_{\alpha}=\operatorname{span}\left\{x^{\alpha}\right\}, \alpha \in \mathbb{N}_{0}^{s}$, and the $\mathrm{H}$ ("homogeneous") gradings, where $\Gamma=\mathbb{N}_{0}$ with the natural order and

$$
\Pi_{n}=\operatorname{span}\left\{x^{\alpha}:|\alpha|=n\right\}, \quad n \in \mathbb{N}_{0} .
$$

Since any polynomial $f \in \Pi$ can be uniquely expanded into the finite sum

$$
f=\sum_{\gamma \in I(f)} f_{\gamma}, \quad f_{\gamma} \in \Pi_{\gamma}, \quad I(f) \subset \Gamma, \quad \# I(f)<\infty,
$$

of its homogeneous components, we can define the $\Gamma$-degree

$$
\delta_{\Gamma}(f)=\max \left\{\gamma \in I(f): f_{\gamma} \neq 0\right\}
$$

and the $\Gamma$-leading term

$$
\Lambda_{\Gamma}(f)=f_{\delta_{\Gamma}(f)}
$$

of $f$. With this notion of degree at hand, we can define "good" bases for polynomial ideals which are a joint generalization of Gröbner bases and H-bases; cf. [12, 13. Recall that a basis of an ideal $\mathcal{I}$ is a (finite) set $\mathcal{P} \subset \Pi$ such that $\mathcal{I}$ is the ideal generated by $\mathcal{P}$, that is,

$$
\mathcal{I}=\langle\mathcal{P}\rangle:=\left\{\sum_{p \in \mathcal{P}} g_{p} p: g_{p} \in \Pi, p \in \mathcal{P}\right\},
$$

and that Hilbert's Basissatz says that every polynomial ideal has such a finite basis. For practical applications, however, bases with additional properties are preferred.

Definition 2.1. A finite set $\mathcal{G} \subset \Pi$ is called a $\Gamma$-basis (for the ideal $\mathcal{I}=\langle\mathcal{G}\rangle$ ) if any $f \in \mathcal{I}$ can be written as

$$
f=\sum_{g \in \mathcal{G}} f_{g} g, \quad f_{g} \in \Pi, \quad \delta_{\Gamma}\left(f_{g} g\right) \leq \delta_{\Gamma}(f) .
$$

Equivalently, $\mathcal{G}$ is a $\Gamma$-basis if and only if the homogeneous ideal $\Lambda_{\Gamma}(\langle\mathcal{G}\rangle)$ (keep in mind that "homogeneous ideal" means that closure under addition is only requested for homogeneous polynomials from the same $\Pi_{\gamma}$ ) has the form

$$
\Lambda_{\Gamma}(\langle\mathcal{G}\rangle)=\left\langle\Lambda_{\Gamma}(\mathcal{G})\right\rangle_{h}=\bigcup_{\gamma \in \Gamma} V_{\gamma}(\mathcal{G}),
$$

where, for $\gamma \in \Gamma$, the homogeneous space of degree $\gamma$ spanned by the leading terms in $\mathcal{G}$ is defined as

$$
V_{\gamma}(\mathcal{G})=\sum_{g \in \mathcal{G}} \Pi_{\gamma-\delta_{\Gamma}(g)} \Lambda_{\Gamma}(g)=\left\{\sum_{g \in \mathcal{G}} f_{g} g: \delta_{\Gamma}\left(f_{g} g\right)=\gamma\right\} \subseteq \Pi_{\gamma} .
$$


In what follows, we will assume that $\Gamma$ is a monomial grading, that is, all the homogeneous spaces $\Pi_{\gamma}, \gamma \in \Gamma$, are spanned by monomials, and that $\Gamma$ is a strict grading, that is, $\Pi_{0}=\mathbb{C}$. Moreover, we introduce the inner product

$$
(f, g)=(\bar{f}(D) g)(0)
$$

which has been of great use in the study of the least interpolant introduced and investigated by de Boor et al. [2] 3], partially because of the convenient identity

$$
(f, q(D) g)=(f \bar{q}, g), \quad f, g, q \in \Pi \text {. }
$$

Note that, under the assumption of a monomial grading, the inner product (2.4) separates degrees, that is,

$$
\left(\Pi_{\gamma}, \Pi_{\gamma^{\prime}}\right)=0, \quad \gamma \neq \gamma^{\prime} .
$$

The following two results have been proven in [16] for the $\mathrm{H}$-grading and in [15] for the G-grading; the general argument is an almost literal replication of the one in [16].

Theorem 2.2. If $\mathcal{G} \subset \Pi$ is a $\Gamma$-basis, then there exists, for any polynomial $f \in \Pi$, a unique polynomial $r \in \Pi, \delta_{\Gamma}(r) \leq \delta_{\Gamma}(f)$, such that

$$
f=\sum_{g \in \mathcal{G}} f_{g} g+r, \quad \delta_{\Gamma}\left(f_{g} g\right) \leq \delta_{\Gamma}(f), \quad g \in \mathcal{G},
$$

and

$$
r_{\gamma} \perp V_{\gamma}(\mathcal{G}), \quad \gamma \in \Gamma .
$$

The "remainder of division" $r$, denoted by $f \underset{\mathcal{G}}{\overrightarrow{\text { }}}$, will be called the normal form of $f$ with respect to $\mathcal{G}$. It is worthwhile to mention that the uniqueness of $r$ in Theorem 2.2 relies on $\mathcal{G}$ being a $\Gamma$-basis.

Theorem 2.3 ([16]). Let $\Theta$ be a finite set of linearly independent functionals which define an ideal interpolation scheme. With the scalar product (2.4), a monomial

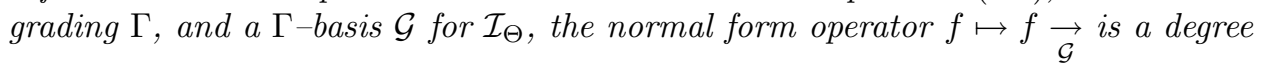
reducing linear interpolation operator and the respective interpolation space satisfies

$$
\Pi \underset{\mathcal{G}}{\longrightarrow}=\bigcap_{g \in \mathcal{G}} \operatorname{ker} \Lambda_{\Gamma}(g)(D) .
$$

Besides the fact that in the case of the $\mathrm{H}$-grading the polynomial spaces defined in equation (2.9) play an important role in box spline theory (cf. [6]), it gives exactly the least interpolant for this particular grading; see [3. However, this identity even yields a simple and useful method to check if a given ideal basis is a $\Gamma$-basis.

Theorem 2.4. Let $\mathcal{G}$ be a finite set of polynomials and suppose that $\langle\mathcal{G}\rangle$ is zerodimensional. Then $\mathcal{G}$ is a $\Gamma$-basis if and only if

$$
\operatorname{dim}\left(\bigcap_{g \in \mathcal{G}} \operatorname{ker} \Lambda_{\Gamma}(g)(D)\right)=\operatorname{dim}(\Pi /\langle\mathcal{G}\rangle) .
$$

Proof. We first remark that the zero-dimensionality of $\langle\mathcal{G}\rangle$ implies that there exists a set $\Theta \in \Pi^{\prime}$ of linearly independent functionals such that $\langle\mathcal{G}\rangle=\operatorname{ker} \Theta$ and, in particular,

$$
\# \Theta=\operatorname{dim}(\Pi /\langle\mathcal{G}\rangle)=\operatorname{dim}(\Pi \underset{\mathcal{G}}{\vec{G}})
$$


The necessity of (2.10) then follows directly from Theorem 2.3

For the converse, we decompose each homogeneous subspace into

$$
\Pi_{\gamma}=V_{\gamma}(\mathcal{G}) \oplus W_{\gamma}(\mathcal{G}), \quad \gamma \in \Gamma,
$$

and note that $\operatorname{dim} V_{\gamma}(\mathcal{G}) \leq \operatorname{dim}\left(\langle\mathcal{G}\rangle \cap \Pi_{\gamma}\right)$, hence, since $\Pi=\Pi /\langle\mathcal{G}\rangle+\langle\mathcal{G}\rangle$,

$$
\operatorname{dim} W_{\gamma} \geq \operatorname{dim}\left(\Pi /\langle\mathcal{G}\rangle \cap \Pi_{\gamma}\right), \quad \gamma \in \Gamma,
$$

with equality if and only if $V_{\gamma}(\mathcal{G})=\left(\Lambda(\langle\mathcal{G}\rangle) \cap \Pi_{\gamma}\right)$ for all $\gamma \in \Gamma$, that is, if and only if $\mathcal{G}$ is a $\Gamma$-basis. Using the identity

$$
W_{\gamma}(\mathcal{G}) \subseteq \bigcap_{g \in \mathcal{G}} \operatorname{ker} \Lambda_{\Gamma}(g)(D)
$$

and assuming that $\mathcal{G}$ is no $\Gamma$-basis, we then obtain from (2.11) that

$\operatorname{dim}\left(\bigcap_{g \in \mathcal{G}} \operatorname{ker} \Lambda_{\Gamma}(g)(D)\right) \geq \sum_{\gamma \in \Gamma} \operatorname{dim} W_{\gamma}(\mathcal{G})>\sum_{\gamma \in \Gamma} \operatorname{dim}\left(\Pi /\langle\mathcal{G}\rangle \cap \Pi_{\gamma}\right)=\operatorname{dim}(\Pi /\langle\mathcal{G}\rangle)$, which completes the proof of the theorem.

It remains to verify (2.12). To this end, we first recall that the monomials form an orthogonal basis for $\Pi$, and that, for any $f \in \Pi$,

$$
f=\sum_{\alpha \in \mathbb{N}_{0}^{s}}\left(x^{\alpha}, f\right) \frac{x^{\alpha}}{\alpha !} .
$$

Therefore, for any $g \in \mathcal{G}$ and $f \in \Pi$ we have that

$$
\Lambda_{\Gamma}(g)(D) f=\sum_{\alpha \in \mathbb{N}_{0}^{s}}\left(x^{\alpha}, \Lambda_{\Gamma}(g)(D) f\right) \frac{x^{\alpha}}{\alpha !}=\sum_{\alpha \in \mathbb{N}_{0}^{s}}\left(x^{\alpha} \Lambda_{\Gamma}(\bar{g}), f\right) \frac{x^{\alpha}}{\alpha !},
$$

and consequently $f \in W_{\gamma}(\mathcal{G})$ implies that $\Lambda_{\Gamma}(g)(D) f=0$, which proves (2.12).

From the proof of Theorem 2.4 we can also draw the following conclusion.

Corollary 2.5. Let $\mathcal{G} \subset \Pi$ be a finite set of polynomials. Then

$$
\operatorname{dim}(\Pi /\langle\mathcal{G}\rangle) \leq \operatorname{dim}\left(\bigcap_{g \in \mathcal{G}} \operatorname{ker} \Lambda_{\Gamma}(g)(D)\right)
$$

with equality if and only if $\mathcal{G}$ is a $\Gamma$-basis.

\section{ZERO CONDITIONS}

Let $E_{s}=\left\{e \in \mathbb{R}^{s}: e_{j}= \pm 1, j=1, \ldots, s\right\}$ denote the set of edges of the cube $[-1,1]^{s}$ and set $Z_{s}:=E_{s} \backslash\{\mathbf{1}\}$, where $\mathbf{1}=(1, \ldots, 1)$. We start by giving a (universal) $\Gamma$-basis for the ideal

$$
\mathcal{J}_{n}=\left\{f \in \Pi:(q(D) f)\left(E_{s}\right)=0, q \in \Pi_{n}\right\}, \quad n \in \mathbb{N}_{0},
$$

determined by the fat points of order $n$ of $Z_{s}$. It is obvious that $\mathcal{J}_{n}$ is an ideal for $n \in \mathbb{N}_{0}$. Moreover, $\mathcal{J}_{0}$ has already been identified in [4] as

$$
\mathcal{J}_{0}=\left\langle z_{1}^{2}-1, \ldots, z_{s}^{2}-1\right\rangle .
$$

We use an abbreviated notation from now on: we use $z-1$ for the vector $\left(z_{1}-1, \ldots\right.$, $\left.z_{s}-1\right)$ and, in the same way, we will write $z^{2}-1=\left(z_{1}^{2}-1, \ldots, z_{s}^{2}-1\right)$. In this notation, equation (3.2) becomes $\mathcal{J}_{0}=\left\langle z^{2}-1\right\rangle$. The first result is as follows. 
Theorem 3.1. For $n \in \mathbb{N}_{0}$ we have that

$$
\mathcal{J}_{n}=\left\langle\left(z^{2}-1\right)^{\alpha}:|\alpha|=n+1\right\rangle,
$$

where $\left\{\left(z^{2}-1\right)^{\alpha}:|\alpha|=n+1\right\}$ is a $\Gamma$-basis for any strict monomial grading $\Gamma$.

Proof. The inclusion

$$
\left\langle\left(z^{2}-1\right)^{\alpha}:|\alpha|=n+1\right\rangle \subseteq \mathcal{J}_{n}
$$

is easily checked by straightforward computations.

To verify the converse inclusion, we denote by $\mathcal{J}=\left\langle\left(z^{2}-1\right)^{\alpha}:|\alpha|=n+1\right\rangle$ the ideal generated by our candidates for a basis and claim that the polynomials

$$
\mathcal{F}=\left\{\left(z^{2}-1\right)^{\alpha}:|\alpha|=n+1\right\}
$$

are a $\Gamma$-basis for $\mathcal{J}$ for any strict monomial grading $\Gamma$. For this purpose, we first note that $\Gamma$ being a strict monomial grading implies, for any $j=1, \ldots, s$, that $\Lambda_{\Gamma}\left(z_{j}^{2}-1\right)=z_{j}^{2}$ and since, for any $p, q \in \Pi$, we have $\Lambda_{\Gamma}(p q)=\Lambda_{\Gamma}(q) \Lambda_{\Gamma}(q)$, it follows that

$$
\Lambda_{\Gamma}\left(\left(z^{2}-1\right)^{\alpha}\right)=z^{2 \alpha}, \quad \alpha \in \mathbb{N}_{0}^{s}
$$

Consequently,

$$
\bigcap_{f \in \mathcal{F}} \operatorname{ker} \Lambda_{\Gamma}(f(D))=\bigcap_{|\alpha|=n+1} \operatorname{ker} D^{2 \alpha}=\bigcap_{|\alpha|=n+1} \operatorname{span}\left\{z^{\beta}: \beta \leq 2 \alpha, \beta \neq 2 \alpha\right\} .
$$

Now, we choose any such $\beta \in \mathbb{N}_{0}^{s}$,

$$
\beta \notin\{2 \alpha:|\alpha|=n+1\} \quad \text { and } \quad \beta \leq 2 \alpha, \quad|\alpha|=n+1,
$$

write $\beta$ as $\beta=2 \gamma+\epsilon, \epsilon \in\{0,1\}^{s}$, and obtain that, for any $\alpha \in \mathbb{N}_{0}^{s},|\alpha|=n+1$, we have that $\gamma \leq \alpha$ and $\gamma \neq \alpha$. This, however, is equivalent to $|\gamma| \leq n$. Hence,

$$
\bigcap_{|\alpha|=n+1}\left\{z^{\beta}: \beta \leq 2 \alpha\right\} \subseteq \bigcup_{\epsilon \in\{0,1\}^{s}}\left\{z^{2 \beta+\epsilon}:|\beta| \leq n\right\},
$$

and so we conclude that

$$
\operatorname{dim}\left(\bigcap_{f \in \mathcal{F}} \operatorname{ker} \Lambda_{\Gamma}(f(D))\right) \leq 2^{s}\left(\begin{array}{c}
n+s \\
s
\end{array}\right) .
$$

On the other hand, the number of linearly independent interpolation conditions which have to be annihilated by $\mathcal{J}_{n}$ and thus the dimension of $\Pi / \mathcal{J}_{n}$ is also $2^{s}\left(\begin{array}{c}n+s \\ s\end{array}\right)$ and therefore there must be equality in (3.8) and hence in (3.7), too. By Theorem 2.4, this implies that $\mathcal{F}$ is a $\Gamma$-basis for $\mathcal{J}_{n}$.

For any two ideals $\mathcal{I}, \mathcal{J} \subseteq \Pi$, their quotient $\mathcal{I}: \mathcal{J}$ is defined as

$$
\mathcal{I}: \mathcal{J}=\{f \in \Pi: f \mathcal{J} \subseteq \mathcal{I}\} .
$$

It is well known (cf. [5]) that $\mathcal{I}: \mathcal{J}$ is again an ideal and that $\mathcal{I} \subseteq \mathcal{I}: \mathcal{J}$. Geometrically, the operation of taking ideal quotients corresponds to "subtraction" of the associated varieties. In view of this relationship, the following result is not very surprising. 
Theorem 3.2. For $n \in \mathbb{N}_{0}$ we have that

$$
\mathcal{I}_{n}:=\left\{f \in \Pi: q(D) f\left(Z_{s}\right)=0, q \in \Pi_{n}\right\}=\left\langle z^{2}-1\right\rangle^{n+1}:\langle z-1\rangle^{n+1} .
$$

Proof. We write

$$
\mathcal{J}=\left\{f \in \Pi: q(D) f\left(Z_{s}\right)=0, q \in \Pi_{n}\right\}
$$

and first note that

$$
\langle z-1\rangle^{n+1} \mathcal{J} \subseteq \mathcal{J}_{n}=\left\langle z^{2}-1\right\rangle^{n+1},
$$

hence $\mathcal{J} \subseteq\left\langle z^{2}-1\right\rangle^{n+1}:\langle z-1\rangle^{n+1}$. For the converse inclusion, we pick any $f \in \mathcal{J}_{n}:\langle z-1\rangle^{n+1}$ and claim that

$$
\frac{\partial^{|\alpha|} f}{\partial z^{\alpha}}\left(Z_{s}\right)=0, \quad \alpha \in \mathbb{N}_{0}^{s},|\alpha| \leq n
$$

We prove (3.11) by induction on $k:=|\alpha|$. In the case $k=0$ we choose $\zeta \in Z_{s}$ and note that there exists at least one index $j \in\{1, \ldots, s\}$ such that $\zeta_{j}=-1$. Therefore,

$$
0=\left(\left(z_{j}-1\right)^{n+1} f\right)(\zeta)=(-2)^{n+1} f(\zeta),
$$

hence $f\left(Z_{s}\right)=0$. Now, suppose that (3.11) has been verified for all $|\alpha| \leq k$, where $0 \leq k<n$. Choose $\alpha \in \mathbb{N}_{0}^{s},|\alpha|=k+1$, as well as $\zeta \in Z_{s}$ and assume again that $\zeta_{j}=-1$. Now we obtain, using the Leibniz formula and the induction hypothesis, that

$$
\begin{aligned}
0 & =\frac{\partial^{k+1}}{\partial z^{\alpha}}\left(\left(z_{j}-1\right)^{n+1} f\right)(\zeta)=\sum_{\eta \leq \alpha}\left(\begin{array}{l}
\alpha \\
\eta
\end{array}\right) \frac{\partial^{|\alpha-\eta|}\left(z_{j}-1\right)^{n+1}}{\partial z^{\alpha-\eta}}(\zeta) \frac{\partial^{|\eta|} f}{\partial z^{\eta}}(\zeta) \\
& =(-2)^{n+1} \frac{\partial^{|\alpha|} f}{\partial z^{\alpha}}(\zeta),
\end{aligned}
$$

which advances the induction hypothesis and thus proves (3.11). Since (3.11) shows that $\mathcal{J} \supseteq\left\langle z^{2}-1\right\rangle^{n+1}:\langle z-1\rangle^{n+1}$, the proof is complete.

Remark 3.3. 1. For $n=0$ Theorem 3.2 follows directly from the well-known fact (cf. [5. Theorem 7, p. 193]) that over algebraically closed fields the variety associated to an ideal quotient $\mathcal{I}: \mathcal{J}$, where $\mathcal{I}$ is radical, equals the (Zariski closure of) the difference. In contrast to this general result, Theorem 3.2 treats only zerodimensional ideals, but includes multiplicities. Nevertheless, this fact might be known or folklore; however, we include it here for the sake of completeness.

2 . It is easy to see that the (primary) ideal $\langle z-1\rangle^{n}$ can be written as

$$
\langle z-1\rangle^{n}=\left\langle(z-1)^{\alpha}:|\alpha|=n\right\rangle, \quad n \in \mathbb{N},
$$

and that the representation on the right-hand side is again a $\Gamma$-basis for any strict monomial grading $\Gamma$. Therefore, it is possible to compute Gröbner bases for the ideal $\left\langle z^{2}-1\right\rangle^{n+1}:\langle z-1\rangle^{n+1}$ explicitly, for example by the method proposed in [5, p. 195].

3. For $s=1$ the quotient ideal is

$$
\left\langle z^{2}-1\right\rangle^{n+1}:\langle z-1\rangle^{n+1}=\langle z+1\rangle^{n+1}
$$

which precisely recovers the factorizations from (1.4). 
Now suppose that $a \in \mathcal{I}_{n}$. Then the above results yield the existence of a polynomial matrix

$$
\boldsymbol{B}=\left[b_{\alpha, \beta}:|\alpha|=|\beta|=n+1\right]
$$

such that

$$
(z-1)^{\alpha} a(z)=\sum_{|\beta|=n+1} b_{\alpha, \beta}(z)\left(z^{2}-1\right)^{\beta}, \quad|\alpha|=n+1,
$$

and since both $(z-1)^{\alpha}$ and $\left(z^{2}-1\right)^{\beta}$ are $\Gamma$-bases for the ideal they generate as long as the grading is a strict monomial one, we can conclude that

$$
\delta_{\Gamma}\left(b_{\alpha, \beta}\right)<\delta_{\Gamma}(a), \quad|\alpha|=|\beta|=n+1,
$$

so as in the univariate situation factorizing is degree-reducing. It is also worthwhile to remark that the matrix (3.12) plays a crucial role in "differencing" the subdivision operator $S_{a}$ which acts on (scalar valued) sequences $c=\left(c_{\alpha} \in \mathbb{R}: \alpha \in \mathbb{Z}^{s}\right)$ as

$$
S_{a} c=\left(\sum_{\beta \in \mathbb{Z}} a_{\alpha-2 \beta} c_{\beta}: \alpha \in \mathbb{Z}\right)
$$

It has been pointed out in [4, p. 57ff] and also in [10] that the case $n=0$ in (3.13) corresponds to the decomposition $\Delta S_{a}=S_{B} \Delta$, where

$$
\Delta c=\left[c_{\alpha+\varepsilon j}-c_{\alpha}: j=1, \ldots, s\right]
$$

denotes the forward difference operator or discrete gradient. Here, the subdivision operator $S_{B}$ acts on vector valued sequences. Therefore our above observations yield that $\alpha \in \mathcal{I}_{n}$ implies that there exist matrix valued polynomials $\boldsymbol{B}_{1}, \ldots, \boldsymbol{B}_{n+1}$ such that

$$
\Delta^{k} S_{a}=S_{\boldsymbol{B}_{k}} \Delta^{k}, \quad k=1, \ldots, n+1, \quad \delta_{\Gamma}\left(\boldsymbol{B}_{n+1}\right)<\cdots<\delta_{\Gamma}\left(\boldsymbol{B}_{1}\right)<\delta_{\Gamma}(a) .
$$

A simpler and more direct way to obtain polynomials in $\left\langle z^{2}-1\right\rangle^{n+1}:\langle z-1\rangle^{n+1}$ is to represent this ideal in another slightly different form. For that end, recall that the product $\mathcal{I} \cdot \mathcal{J}$ of two ideals $\mathcal{I}, \mathcal{J} \subseteq \Pi$ is defined as the ideal generated by all products $f g, f \in \mathcal{I}, g \in \mathcal{J}$.

Corollary 3.4. For $n \in \mathbb{N}_{0}$ we have that

$$
\mathcal{I}_{n}=\left\langle z^{2}-1\right\rangle^{n+1}:\langle z-1\rangle^{n+1}=\left(\left\langle z^{2}-1\right\rangle:\langle z-1\rangle\right)^{n+1}=\mathcal{I}_{0}^{n+1}
$$

Since

$$
\left\langle z^{2}-1\right\rangle=\bigcap_{\zeta \in\{0,1\}^{s}}\langle z-\zeta\rangle=\left(\bigcap_{\zeta \in\{0,1\}^{s} \backslash\{\mathbf{1}\}}\langle z-\zeta\rangle\right) \cap\langle z-1\rangle,
$$

Corollary 3.4 follows directly from Theorem 3.2 and Lemma 3.5 which will be given immediately. For that end, recall that an ideal $\mathcal{I}$ is called prime if $f g \in \mathcal{I}$ and $g \notin \mathcal{I}$ imply that $f \in \mathcal{I}$ and note that all the ideals on the right-hand side are in fact prime ideals.

Lemma 3.5. Let $\mathcal{I}$ be the intersection of a finite number of prime ideals with the ideal $\mathcal{J}$. Then, for any $n \in \mathbb{N}$,

$$
(\mathcal{I}: \mathcal{J})^{n}=\mathcal{I}^{n}: \mathcal{J}^{n} .
$$


Proof. The inclusion " $\subseteq$ " even holds for any pair $\mathcal{J}, \mathcal{J}$ of ideals. Using the two well-known identities $(\overline{\mathcal{I}} \cdot \mathcal{J}): \mathcal{K} \supseteq \mathcal{I} \cdot(\mathcal{J}: \mathcal{K})$ and $(\mathcal{I}: \mathcal{J}): \mathcal{K}=\mathcal{I}:(\mathcal{J}: \mathcal{K})$ (cf. [14, p. 63]), we obtain this fact by induction on $n$, noting that

$$
\begin{aligned}
\mathcal{I}^{n+1}: \mathcal{J}^{n+1} & =\left(\mathcal{I}^{n+1}: \mathcal{J}^{n}\right): \mathcal{J} \supseteq\left(\mathcal{I} \cdot\left(\mathcal{I}^{n}: \mathcal{J}^{n}\right)\right): \mathcal{J} \supseteq\left(\mathcal{I}^{n}: \mathcal{J}^{n}\right):(\mathcal{I}: \mathcal{J}) \\
& =(\mathcal{I}: \mathcal{J})^{n+1} .
\end{aligned}
$$

For the converse inclusion, " $\supseteq$ ", we first show that (3.16) holds true when $\mathcal{I}$ is a prime ideal. In fact, this is trivial if $\mathcal{I}=\mathcal{J}$ and otherwise it follows by the above argument from the identity

$$
\mathcal{I}^{2}: \mathcal{J}=\mathcal{I} \cdot(\mathcal{I}: \mathcal{J}), \quad \mathcal{I} \text { prime }, \quad \mathcal{J} \neq \mathcal{I},
$$

which we are going to prove first. Since the inclusion " $\supseteq$ " holds in (3.17) for any pair of ideals, we only have to verify " $\subseteq$ ". To that end we recall that, by definition, any $f \in \mathcal{I}: \mathcal{J}$ has to satisfy $f g \in \mathcal{I}$ for any $g \in \mathcal{J}$. Choosing $0 \neq g \in \mathcal{J} \backslash \mathcal{I}$, which is possible since $\mathcal{I} \neq \mathcal{J}$, and the assumption that $\mathcal{I}$ is prime then yields that $f \in \mathcal{I}$, hence $\mathcal{I}: \mathcal{J}=\mathcal{I}$ and therefore $\mathcal{I} \cdot(\mathcal{I}: \mathcal{J})=\mathcal{I}^{2}$. In a similar fashion, suppose that $f \in \mathcal{I}^{2}: \mathcal{J}$, that is, $f g \in \mathcal{I}^{2}, g \in \mathcal{J}$, and choosing $g \in \mathcal{J} \backslash \mathcal{I}$ we now obtain that $f \in \mathcal{I}^{2}$ which proves (3.17).

To finish the proof of (3.16), we use the non-redundant representation

$$
\mathcal{I}=\mathcal{I}_{1} \cap \cdots \cap \mathcal{I}_{N} \cap \mathcal{J}
$$

of $\mathcal{I}$ as an intersection of the different prime ideals $\mathcal{I}_{1}, \ldots, \mathcal{I}_{N}$ with $\mathcal{J}$. Setting $\mathcal{I}_{N+1}=\mathcal{J}$ and making use of $\mathcal{I}_{j}: \mathcal{J}=\mathcal{I}_{j}, j=1, \ldots, N$, we observe that

$$
\mathcal{I}: \mathcal{J}=\bigcap_{j=1}^{N+1}\left(\mathcal{I}_{j}: \mathcal{J}\right)=\bigcap_{j=1}^{N}\left(\mathcal{I}_{j}: \mathcal{J}\right) \cap(\mathcal{J}: \mathcal{J})=\bigcap_{j=1}^{N}\left(\mathcal{I}_{j}: \mathcal{J}\right)=\bigcap_{j=1}^{N} \mathcal{I}_{j},
$$

hence, by (3.17),

$$
\begin{aligned}
(\mathcal{I}: \mathcal{J})^{n} & =\left(\bigcap_{j=1}^{N} \mathcal{I}_{j}\right)^{n}=\bigcap_{j=1}^{N} \mathcal{I}_{j}^{n}=\bigcap_{j=1}^{N}\left(\mathcal{I}_{j}: \mathcal{J}\right)^{n}=\bigcap_{j=1}^{N} \mathcal{I}_{j}^{n}: \mathcal{J}^{n}=\bigcap_{j=1}^{N+1} \mathcal{I}_{j}^{n}: \mathcal{J}^{n} \\
& =\left(\bigcap_{j=1}^{N+1} \mathcal{I}_{j}^{n}\right): \mathcal{J}^{n}=\mathcal{I}^{n}: \mathcal{J}^{n} .
\end{aligned}
$$

Corollary 3.4 is useful for the practical determination of "masks that yield approximation power", for example by means of a computer algebra program. Provided that we know a basis of $\mathcal{I}_{0}$, which will be given in Proposition 4.1immediately, we can construct a first basis of $\mathcal{I}_{n}=\mathcal{I}_{0}^{n+1}$ by "simply" taking all products of $n+1$ elements of the basis of $\mathcal{I}_{0}$, repetitions allowed. Of course, such a highly redundant basis which consists of $(s+1)^{n+1}$ elements can be reduced to a "simpler" ideal basis automatically by computing the associated Gröbner basis, preferably with a term 
order subordinate total degree. Such a basis, let us call it $\mathcal{G}_{n}$, can now be used in various ways:

1. To "parameterize" all potential masks with desired approximation power by considering the set of polynomials

$$
\left\{\sum_{g \in \mathcal{G}_{n}} p_{g} g: p_{g} \in \Pi\right\} \text {. }
$$

The coefficients of these $p_{g}$ then define an associated joint spectral radius which one might try to adjust by some standard optimization routines in order to obtain refinable functions.

2. To give estimates on the minimal support of such masks in terms of the total degree. If a Gröbner basis has been computed with respect to a term order which is subordinate to total degree, then any polynomial in the ideal must have at least the same total degree as the least degree element in the basis.

However, the use of Gröbner bases will usually destroy symmetry or other structural information provided by the quotient ideal. For example, the representation (4.2) cannot be obtained by symbolic manipulations.

\section{Two EXAmples}

We end the paper by deriving and discussing explicit descriptions for $\mathcal{I}_{0}$ and $\mathcal{I}_{1}$. The first one, $\mathcal{I}_{0}$, is very simple, indeed.

Proposition 4.1 (Description of $\mathcal{I}_{0}$ ).

$$
\mathcal{I}_{0}=\left\langle z^{2}-1\right\rangle:\langle z-1\rangle=\left\langle z^{2}-1\right\rangle+\left\langle\left(z_{1}+1\right) \cdots\left(z_{s}+1\right)\right\rangle .
$$

Note that the additional polynomial $\left(z_{1}+1\right) \cdots\left(z_{s}+1\right)$ corresponds to the mask of the characteristic function of the unit cube $[0,1]^{s}$, which is of course refinable.

Proof. Set

$$
\mathcal{G}=\left\{z_{1}^{2}-1, \ldots, z_{s}^{2}-1,\left(z_{1}+1\right) \cdots\left(z_{s}+1\right)\right\} .
$$

Clearly, $\mathcal{G}\left(Z_{s}\right)=0$, hence $\langle\mathcal{G}\rangle \subseteq\left\langle z^{2}-1\right\rangle:\langle z-1\rangle$. Moreover, for any strict monomial grading $\Gamma$ we have that $\Lambda_{\Gamma}(\mathcal{G})=\left\{z_{1}^{2}, \ldots, z_{s}^{2}, z_{1} \cdots z_{s}\right\}$. Thus,

$$
\operatorname{dim}\left(\bigcap_{f \in \Lambda_{\Gamma}(\mathcal{G})} f(D)\right)=2^{s}-1=\operatorname{dim}\left(\Pi /\left(\left\langle z^{2}-1\right\rangle:\langle z-1\rangle\right)\right),
$$

and so, by Theorem [2.4 $\mathcal{G}$ is even a $\Gamma$-basis for $\left\langle z^{2}-1\right\rangle:\langle z-1\rangle$.

Proposition 4.2 (Description of $\mathcal{I}_{1}$ ).

$$
\begin{aligned}
\mathcal{I}_{1} & =\left\langle z^{2}-1\right\rangle^{2}:\langle z-1\rangle^{2} \\
& =\left\langle\left(z^{2}-1\right)^{\alpha}:|\alpha|=2\right\rangle+\left\langle\left(z_{1}+\cdots+z_{s}-(s-2)\right)\left(z_{1}+1\right) \cdots\left(z_{s}+1\right)\right\rangle,
\end{aligned}
$$

and the polynomials on the right-hand side of equation (4.2) form an $\mathrm{H}$-basis for the ideal $\mathcal{I}_{1}$. 
Before getting to the proof of this result two remarks are appropriate. First, it is important to note that the basis from (4.2) is no Gröbner basis for any term order, not even a term order which is subordinate to the total degree. This can be verified by combining Theorem [2.4 with a simple counting argument and shows that indeed the concept of an $\mathrm{H}$-basis provides a significantly improved generality in comparison to Gröbner bases with respect to a term order subordinate to total degree which nevertheless yields an $\mathrm{H}$-basis, too. Second, the "additional" basis function in (4.2) has an appealing geometric interpretation for $s=2$ : the unique minimally supported refinable function (in the sense of total degree) is the one according to the mask

$$
a(z)=\frac{z_{1}+z_{2}}{2}\left(z_{1}+1\right)\left(z_{2}+1\right)
$$

which is the well-known (piecewise linear) three directions box spline. This function is piecewise linear on the triangulation of its support which is given by the three directions $e_{1}, e_{2}, e_{1}+e_{2}$; see Figure 1 For higher dimensions, however, I am not aware of geometric interpretation of the mask

$$
\frac{z_{1}+\cdots+z_{s}-s+2}{2} \prod_{j=1}^{s}\left(z_{j}+1\right) .
$$

Moreover, since this polynomial has degree $s+1 \geq 4$ if $s \geq 3$, the minimally supported refinable function of approximation order 1 is no more unique since we can always add multiples of the polynomials $\left(z^{2}-1\right)^{\alpha},|\alpha|=2$, without increasing the degree.

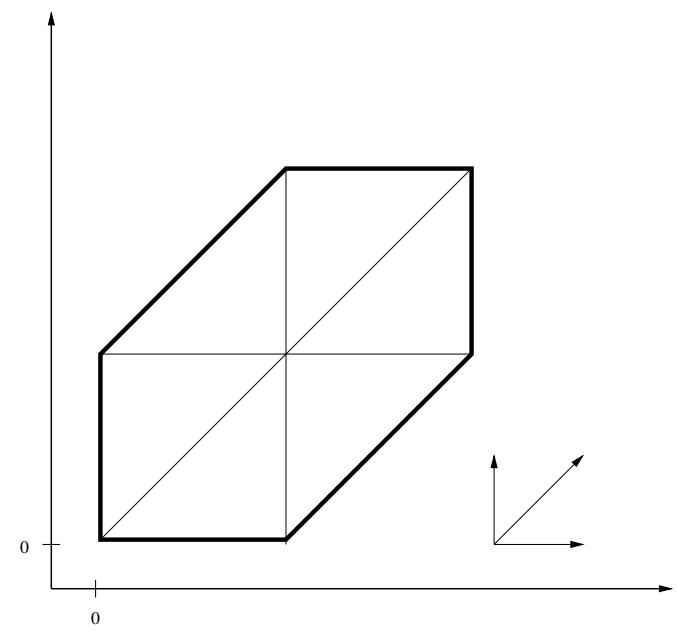

FiguRE 1. The three directions box spline and the three directions.

Also observe that the normalization condition (1.6), that is, $a(\mathbf{1})=1$, requests that the polynomial $a$ must always contain a nontrivial contribution by the "additional polynomials" connected to the characteristic function of the unit cube in the case of $\mathcal{I}_{0}$ and to the $(s+1)$-directions "box-spline" in the case of $\mathcal{I}_{1}$, respectively, which yields that the minimal degrees of the mask necessary for approximation order 1 or 2 are $s$ and $s+1$, respectively. 
Proof of Proposition 4.2. Let

$$
\begin{aligned}
\mathcal{F} & =\left\{\left(z^{2}-1\right)^{\alpha}:|\alpha|=2\right\} \cup\left\{\left(z_{1}+\cdots+z_{s}-s+2\right)\left(z_{1}+1\right) \cdots\left(z_{s}+1\right)\right\} \\
& =: \mathcal{G} \cup\{f\}
\end{aligned}
$$

denote the polynomials generating the ideal on the right-hand side of (4.2). It is easy to verify that all these polynomials in $\mathcal{F}$ vanish together with their first derivatives at $Z_{s}$ and so we obtain that $\langle\mathcal{F}\rangle \subseteq \mathcal{I}_{1}$ and thus

$$
\left(2^{s}-1\right)(s-1)=\operatorname{dim}\left(\Pi / \mathcal{I}_{1}\right) \leq \operatorname{dim}(\Pi /\langle\mathcal{F}\rangle) .
$$

Recalling the proof of Theorem 3.1, we also recall that

$$
\operatorname{dim} \mathcal{P}=2^{s}(s+1), \quad \mathcal{P}=\bigcap_{g \in \mathcal{G}} \operatorname{ker} \Lambda(g)(D)=\bigcap_{|\alpha|=2} \operatorname{ker} D^{2 \alpha},
$$

where $\Lambda=\Lambda_{H}$ denotes the homogeneous leading term. However, defining the linearly independent polynomials

$$
f_{j}(z)=z_{j} f(z)-\sum_{k \neq j}\left(z_{j}^{2}-1\right)\left(z_{k}^{2}-1\right), \quad j=1, \ldots, s,
$$

we observe that $f, f_{j} \notin \operatorname{ker} \Lambda(f)(D), j=1, \ldots, s$, while the identities

$$
\Lambda(f)=\sum_{k=1}^{s} z^{\mathbf{1 + e _ { k }}}, \quad \Lambda\left(f_{j}\right)=z^{\mathbf{1 + 2 e _ { j }}}, \quad j=1, \ldots, s,
$$

imply that $f, f_{j} \in \mathcal{P}, j=1, \ldots, s$. Thus, we conclude that

$$
\operatorname{dim}\left(\bigcap_{f \in \mathcal{F}} \operatorname{ker} \Lambda(f)(D)\right) \leq \operatorname{dim}\left(\bigcap_{g \in \mathcal{G}} \operatorname{ker} \Lambda(g)(D)\right)-(s+1)=\left(2^{s}-1\right)(s+1),
$$

which yields, together with (4.3) and Corollary 2.5 that

$$
\left(2^{s}-1\right)(s-1)=\operatorname{dim}\left(\Pi / \mathcal{I}_{1}\right) \leq \operatorname{dim}\left(\bigcap_{f \in \mathcal{F}} \operatorname{ker} \Lambda(f)(D)\right) \leq\left(2^{s}-1\right)(s+1)
$$

and another application of Theorem 2.4 yields that $\mathcal{F}$ is in fact an $\mathrm{H}$-basis for $\mathcal{I}_{1}$.

\section{ACKNOWLEDGMENT}

I am very grateful to H. M. Möller for stimulating discussions, all his valuable suggestions, and, in the first place, for pointing out Lemma 3.5 .

\section{REFERENCES}

[1] G. Birkhoff. The algebra of multivariate interpolation. In C.V. Coffman and G.J. Fix, editors, Constructive Approaches to Mathematical Models, pages 345-363. Academic Press, Inc., 1979. MR 83d:41001

[2] C. de Boor and A. Ron. On multivariate polynomial interpolation. Constr. Approx., 6 (1990), 287-302. MR 91c:41005

[3] C. de Boor and A. Ron. The least solution for the polynomial interpolation problem. Math. Z., 210 (1992), 347-378. MR 93f:41002

[4] A. S. Cavaretta, W. Dahmen, and C. A. Micchelli. Stationary Subdivision, volume 93 (453) of Memoirs of the AMS. Amer. Math. Soc., 1991. MR 92h:65017

[5] D. Cox, J. Little, and D. O'Shea. Ideals, Varieties and Algorithms. Undergraduate Texts in Mathematics. Springer-Verlag, 2nd edition, 1996. MR 97h:13024 
[6] W. Dahmen and C. A. Micchelli. Local dimension of piecewise polynomial spaces, syzygies, and the solutions of systems of partial differential operators. Math. Nachr., 148 (1990), 117136. MR 92i:35029

[7] I. Daubechies. Ten Lectures on Wavelets, volume 61 of CBMS-NSF Regional Conference Series in Applied Mathematics. SIAM, 1992. MR 93e:42045.

[8] B. Han and R.-Q. Jia. Multivariate refinement equations and convergence of subdivision schemes. SIAM J. Math. Anal., (1998), 1177-1199. MR 99f:41018

[9] K. Jetter and G. Plonka. A survey on $L_{2}$-approximation order from shift-invariant spaces. Technical Report SM-DU-441, Gerhard Mercator Universität Gesamthochschule Duisburg, 1999.

[10] V. Latour, J. Müller, and W. Nickel. Stationary subdivision for general scaling matrices. Math. Z., 227 (1998), 645-661. MR 99c:65027]

[11] H. M. Möller. Hermite interpolation in several variables using ideal-theoretic methods. In W. Schempp and K. Zeller, editors, Constructive Theory of Functions of Several Variables, Lecture Notes in Mathematics, pages 155-163, Springer, 1977. MR 58:12087

[12] H. M. Möller and T. Sauer. H-bases for polynomial interpolation and system solving. Advances Comput. Math., 12 (2000), 335-362. MR 2001g:41005

[13] H. M. Möller and T. Sauer. H-bases I: The foundation. In A. Cohen, C. Rabut, and L. L. Schumaker, editors, Curve and Surface fitting: Saint-Malo 1999, pages 325-332, Vanderbilt University Press, 2000.

[14] B. Renschuch. Elementare und praktische Idealtheorie. VEB Deutscher Verlag der Wissenschften, 1976. MR 56:2981

[15] T. Sauer. Polynomial interpolation of minimal degree and Gröbner bases. In B. Buchberger and F. Winkler, editors, Groebner Bases and Applications (Proc. of the Conf. 33 Years of Groebner Bases), volume 251 of London Math. Soc. Lecture Notes, pages 483-494, Cambridge University Press, 1998. MR 2000h:13019

[16] T. Sauer. Gröbner bases, H-bases and interpolation. Trans. Amer. Math. Soc., 353 (2001), 2293-2308. MR 2002b:13035

Lehrstuhl für Numerische Mathematik, Justus-Liebig-Universität Giessen, HeinrichBuff-Ring 44, D-35392 Giessen, Germany

E-mail address: Tomas.Sauer@math.uni-giessen.de 